\title{
NiÑOS, NIÑAS Y ADOLESCENTES: MIGRANTES TRABAJADORES guatemaltecos en la ciudad de Tapachula, Chiapas
}

\author{
Yasmina Areli López Reyes
}

Resumen: Este artículo tiene como objetivo evidenciar cómo los fundamentos básicos de algunas instituciones como la OIT y la UNICEF son constantemente pasados por alto en la cotidianidad, ya que a lo largo de 25 años los niños, niñas y adolescentes (NNA) migrantes guatemaltecos continúan experimentando relaciones de desigualdad social y laboral sin que las instituciones hayan logrado cumplir con sus objetivos. De ahi que se considere la necesidad de plantear un cambio de perspectivas, estrategias y acciones para abordar esta problemática, en la que los NNA tengan posibilidades de integrarse en la organización y participación de las mismas y se tome en cuenta su derecho a decidir sobre las formas y condiciones de trabajo.

Palabras clave: migración, niñez trabajadora, organización y participación social.

Enviado a dictamen: 17 de abril de 2012

Aprobación: 04 de mayo de 2012

Revisiones: 1

Mtra. Yasmina Areli López Reyes, maestra en Antropología social por el CiesasSureste, Universidad Intercultural de Chiapas. Temas de especialización: historia local y cambio social a partir del comercio del café y estudios de religión, específicamente, organización y prácticas organizativas en torno al trabajo pastoral diocesano en Chiapas. Correo electrónico: yasareli@hotmail.com.
Abstract: This article present and analyse how the basics of some institutions like OIT and UNICEF are constantly overlooked in the everyday, as we can see that over 25 years Guatemalan children and adolescents migrants continue to experience social relations of inequality and labor without institutions have succeeded in meeting its objectives. That is the reason that we need to consider the need to propose a change of perspectives, strategies and actions to address this issue, in which children and adolescents have opportunities to join the organization and participation of them, where they will have the opportunity to decide about the ways and working conditions.

Keywords: migration, working children, social organization and participation.

\section{Introducción}

L a migración centroamericana hacia México, ya sea de manera transitoria para llegar a Estados Unidos o para establecerse en ciudades fronterizas, ha sido tema recurrente en distintos estudios académicos. En ellos se han descrito, desde las experiencias de los migrantes durante el recorrido, las remesas, las rutas de tránsito, las condiciones laborales, los diagnósticos de salud (principalmente sexual), 
la situación de trata, de género, (Bronfman, 2004; Villafuerte, 2008; Kaufferet. al., 2002; Rivas, 2009; Olivera; 2008, entre otros). Las instituciones gubernamentales y no gubernamentales también han puesto atención a este fenómeno, con la finalidad de atender las demandas y las denuncias en torno a los derechos humanos que como individuos poseen.

En la mayoría de los estudios se hace referencia sólo a la población masculina, ya que los hombres eran quienes regularmente tendían a desplazarse (Durang y Massey, 2003). Pero, a medida que la situación económica, política, ambiental y social en los países de origen se fue haciendo cada vez más crítica — aunado a que el acto de migrar poco a poco fue institucionalizandose como una práctica natural-, las mujeres y los niños, niñas y adolescentes (NNA) se vieron en la necesidad de iniciar el traslado hacia lugares fuera de su país en busca de mejores condiciones de vida, convirtiéndose en nuevos sujetos de estudio, por lo menos en los últimos años. Cabe señalar que dichos sectores de la población siempre han migrado, sin embargo, esto no era un fenómeno tan constante ni tan masivo como lo es actualmente.

La vulnerabilidad y los riesgos que todos los migrantes enfrentan comienzan a partir del momento en que dejan sus hogares y su país de origen. Si bien, una vez fuera, todos están expuestos a una serie de situaciones desfavorables, se considera que hay niveles en los que tanto la vulnerabilidad como el riesgo son mayores y están determinados por la edad y el género. De ahí que el centro de atención se esté dirigiendo hacia las mujeres y hacia la niñez $z^{1}$ adolescentes migrantes.

Tapachula, en el estado de Chiapas, es una de las ciudades en las que más estudios sociológicos y antropológicos se han llevado a cabo para conocer la problemática que representa la migración einmigración, ${ }^{2}$ especialmente la infantil (Azaola, 2000; Casillas, 2006; Escobar, 2008; Nájera, 2011).

Estos estudios cuentan las variadas situaciones y condiciones de vida que experimentan en un país ajeno al suyo en calidad de indocumentados, las actividades y condiciones laborales en las que los niños y adolescentes se desempeñan, además de los espacios en los que viven y conviven social y culturalmente. Asimismo, se ha puesto de manifiesto la vulnerabilidad de la que son objeto por los peligros que conllevan dichas actividades, por los lugares donde viven, porque son menores de edad y por su situación migratoria, entre otros factores.

En Tapachula, ciudad frontera ${ }^{3}$ con Guatemala, existe un número considerable de población infantil trabajando en diversas actividades y en distintos espacios. La mayoría de esta población proviene de los departamentos ubicados en la franja fronteriza de ese país y su presencia en el lugar, en muchos casos, es temporal aunque hay quienes se han establecido permanentemente. Lo que es un hecho es que este tipo de migración se ha realizado durante décadas, pero por lo menos hasta hace aproximadamente 25 años, comenzó a aparecer información escrita bastante restringida al respecto.

Este artículo tiene el objetivo de evidenciar cómo los fundamentos básicos de algunas instituciones como la OIT y la UNICEF son constantemente pasados por alto en la cotidianidad, ya que podemos ver cómo, a lo largo de 25 años, los NNA migrantes guatemaltecos continúan experimentando relaciones de desigualdad social y laboral sin que las instituciones hayan logrado cumplir con sus objetivos. De ahí que se considere la necesidad de plantear un cambio de perspectivas, estrategias y acciones para abordar esta problemática, en la que los NNA tengan posibilidades de integrarse en la organización y participación de las mismas y se tome en cuenta su derecho a decidir sobre las formas y condiciones de trabajo.

También se busca poner en justa dimensión la realidad que viven los NNA en la ciudad, evitando caer en posicionamientos paternalistas y victimizados que, lejos de favorecerles, les perjudican a tal grado que les quitan la capacidad de agencia necesaria en el discurso 
para enfrentar la violencia y vulnerabilidad de la que son objeto.

Para ello, primero se presenta una revisión bibliográfica sobre las implicaciones que conlleva hablar de trabajo infantil vs niñez trabajadora. Después, se expone un breve recuento histórico acerca de cómo los NNA se fueron insertando en el mercado laboral en la ciudad de Tapachula, así como los cambios y las continuidades existentes en relación a las actividades y a las condiciones laborales en los que la población infantil se ha desarrollado en la ciudad de Tapachula durante un periodo de 25 años. Finalmente, a la luz del dato etnográfico, se plantea la alternativa de fomentar la organización y participación de los niños, niñas y adolescentes como sujetos de acción en beneficio propio.

\section{Trabajo infantil vs. niñez trabajadora}

La literatura que aborda la temática sobre el concepto de trabajo infantil es amplia y ha sido escrita desde diversas áreas de las ciencias sociales (antropología, sociología, psicología). Los estudios realizados por varias instituciones gubernamentales y no gubernamentales también han contribuido a la definición de dicho concepto. Sin embargo, en los últimos años, algunos especialistas en el tema se han dado a la tarea de entender la dinámica laboral de los NNA de una manera distinta y sus resultados han arrojado otras alternativas de estudio en las que ya no se habla de trabajo infantil, sino de niñez trabajadora.

Antes de establecer una diferencia a partir de la comparación entre ambos conceptos, es necesario tener en cuenta a qué se refiere la categoría de niño y adolescente. De acuerdo con la Convención sobre los Derechos del Niño, se entiende por niño "todo ser humano menor de dieciocho años de edad, salvo que, en virtud de la ley que le sea aplicable, haya alcanzado antes la mayoría de edad." ${ }^{4}$

El Artículo 2 de la Ley para la Protección de los Derechos de las Niñas, Niños y Adolescentes, define el término niño en concordancia con el Artículo l de la mencionada Convención y divide la categoría de niño en dos grupos etarios: niñas y niños (hasta los 12 años incompletos) y adolescentes (desde los 13 años cumplidos hasta los 18 años cumplidos). ${ }^{5}$

Sin embargo, es conveniente reconocer que la categoría de niñez y adolescencia parte de una construcción social definida tanto por lo biológico como por "el entrecruzamiento de variables sociales, culturales, psicológicas, económicas y jurídicas que la han definido como una categoría diferenciada del mundo adulto a través de un largo proceso histórico, posicionándola igualmente en la perspectiva de los derechos humanos (Pico y Salazar, 2008: 98).

El concepto de trabajo infantil que se plantea en este artículo parte de dos posicionamientos generales, los cuales no están totalmente contrapuestos: el institucional y el académico-organizativo. En el institucional no están de acuerdo con que desarrollen actividades laborales, si ven afectadas su integridad física, psicológica, social o moral. En contraste, el académico-organizativo, abre la posibilidad de que desempeñen trabajos (oficios) de manera digna, que contribuyan a mejorar su desarrollo personal y su economía familiar, sin vulnerar sus derechos ni su integridad.

Entre quienes están en desacuerdo con el trabajo infantil encontramos principalmente a la Organización Internacional del Trabajo (OIT), a través de la Convención Internacional sobre los Derechos del niño y la niña, y a UNICEF. La OIT define como trabajo infantil "todo trabajo que priva a los niños de su niñez, su potencial y su dignidad, y que es perjudicial para su desarrollo físico y psicológico". Es decir, sería trabajo infantil toda aquella actividad considerada física, mental, social o moralmente peligrosa o nociva para el niño, que interfiera en su proceso de escolarización, ya sea que lo prive de su posibilidad de ir a la escuela, que la abandone prematuramente o que se vea en la necesidad de combinar la asistencia a la escuela con 
altas exigencias relacionadas con un trabajo pesado y jornadas laborales intensivas. ${ }^{6}$

No obstante, la institución habla de trabajo infantil aceptable siempre y cuando no afecte a la salud de los niños, ni a su desarrollo personal y que no interfiera con su escolarización. Además, reconoce que las labores de ayuda a los padres en la casa por parte de los niños, colaborar en la empresa familiar, ganar dinero en vacaciones o fuera del horario escolar para sus propios gastos, es positivo para desarrollar el sentido de responsabilidad, la autoestima, el desarrollo afectivo y de habilidades que no perjudican al niño.

La UNICEF define el trabajo infantil bajo ciertos criterios básicos. Es inapropiado cuando es con dedicación exclusiva a una edad demasiado temprana (menos de 12 años). Demasiadas horas de trabajo, provocan estrés físico, social o psicológico indebido, se trabaja y se vive en la calle en malas condiciones, el salario es inadecuado, el niño tiene que asumir demasiada responsabilidad, el trabajo impide el acceso a la escolarización, el trabajo mina la dignidad y autoestima del niño (como el esclavismo y la explotación sexual), e impide conseguir un pleno desarrollo social y psicológico. ${ }^{7}$

La contraparte de esta forma de entender el trabajo infantil la encontramos en lo que se conoce como cultura o movimientos de los niños, niñas y adolescentes trabajadores (NATs), desde donde se defiende su derecho a decidir si trabajan o no. Consideran a los niños, niñas y adolescentes como sujetos de derechos y no sólo como objetos, es decir, como protagonistas de su vida con capacidad de decidir lo que les conviene o no les conviene. Además, consideran que el problema no es que los NNA trabajen, sino las condiciones en las que realizan esas actividades.

Entre los principales exponentes de este segundo planteamiento encontramos a Alejandro Cussiánovich y a Manfred Liebel. Ambos autores, rechazan la idea de continuar abordando el trabajo de los niños desde una visión que ellos llaman adulto-céntrica (o paternalista), en la que lo que prevalece es la opinión de los adultos sobre lo que es mejor para los NNA.

Cussiánovich afirma que si la OIT hizo excepciones al Convenio 182 se debió a las presiones que realizaron diferentes sectores, entre los que se encuentran "los movimientos de NATs de América Latina, de África y de la India. Para este autor, la declaración en Kundapur en noviembre de 1996, la participación pública de un adolescente peruano trabajador en la conferencia de Ministros de Trabajo de Ámsterdam a inicios de 1997, la posición del Movimiento de NATs de la Región, rechazando el título del texto borrador definido como un convenio por la abolición del trabajo infantil, pero sobre todo, incluir entre las formas de trabajo a la prostitución infantil, al tráfico de menores, los niños soldados ...aunque éstas sean consideradas dentro de las peores formas de trabajo]. llevan a Cussiánovich a la idea de que un niño y adolescente trabajador debe ser 'rehabilitado' luego que deja de ser trabajador. (Cussiánovich, 2002: 9).

También critica el hecho de que los convenios se redacten en torno a la prohibición, la eliminación, la sanción, la penalización del trabajo infantil y no para dar valor y reconocer la vida y el trabajo de los NNA trabajadores. Considera que entender el trabajo como uno de los "ejes catalizadores de las grandes interrogantes" y considerar los cambios que se han generado en torno al concepto, nos obliga a asumir nuevas dimensiones de análisis.

Leibel coincide con los aportes de Cussiánovich. Señala que el paternalismo, al haber prevalecido por décadas, es uno de los principales obstáculos para entender a los NATs como sujetos de acción. Distingue entre el paternalismo tradicional y el paternalismo moderno. El tradicional:

consiste en la subordinación absoluta del niño. De acuerdo con él, los adultos son los únicos que deciden qué es bueno y qué deben hacer los niños. Todas las 
normas están establecidas y no son 'discutibles'. No se les concede a los niños un 'ámbito propio' (Liebel, 2007: 115).

\section{El moderno:}

(...) cede a los niños un 'mundo propio' regido por 'leyes' propias; se les ofrece la posibilidad de desarrollarse como personas responsables y maduras. Este paternalimo moderno debe garantizarse fundamentalmente mediante la protección y la asistencia. A los niños se les reservan determinadas zonas, en las cuales todavía no impera 'la seriedad de la vida' y en las cuales pueden manifestar, hasta cierto grado, una vida y una dignidad propias" (Liebel, 2007: 115).

Éste es el tipo de paternalismo que impera entre las instituciones gubernamentales y organizaciones civiles, ya que se basan en lo establecido en la Convención Internacional sobre los Derechos del niño y la niña (OIT). Liebel señala que, si bien, en dicha convención se plasmaron algunos derechos encaminados a la participación, tales como tener una opinión propia y expresarla libremente o el derecho a reunirse y agruparse libremente, de ningún modo:

(...) toman en cuenta las condiciones especiales en las que viven los niños, en particular los de los sectores populares y, en consecuencia, hace prácticamente imposible que se cumplan sus derechos. Además, parecería que la tarea de salirse del campo del paternalismo se asigna únicamente a los niños. Los adultos quedan facultados, como antes, a escuchar lo que dicen los niños y a tomárselo en serio o no. No están obligados a nada que facilite la participación de los niños (Liebel, 2007: 117).

Liebel considera inútil que se realicen conferencias de adultos preocupados por el cumplimiento de los derechos de los niños, niñas y adolescentes o que estos conozcan esos derechos si se les sigue viendo como un problema más que como un potencial social y se siguen reproduciendo las viejas prácticas paternalistas bajo nuevas versiones.

Por ello, su apuesta está orientada hacia los movimientos de los NATs, los cuales surgen a partir de los debates entre los niños, y dentro de la misma Convención en la década de 1980. Consisten en "organizaciones infantiles que son lideradas por los niños y adolescentes, para tener sus propias estructuras y normas y para desarrollar sus propias ideas, demandas y formas de acción, que emergen de la situación de vida y trabajo de sus actores." (Liebel, 2006, 108). Estas organizaciones pueden tener el apoyo de organizaciones humanitarias adultas o juveniles que les aporten consejos, críticas y generen espacios propios de expresión o les sirvan como meros intermediarios y acompañantes del proceso, mas no deben ser los que tomen las decisiones. El nivel de operación puede ser nacional, aunque generalmente están limitados por profesión u oficio y por los lugares de trabajo.

Aunque estas organizaciones se crearon como medios de expresión de los NATs para exigir sus derechos humanos y laborales, Liebel comenta que no todas reclaman el 'derecho a trabajar', pero sí coinciden en que su trabajo debe ser valorado y no discriminado, además de ser reconocido socialmente ya que, al ver su trabajo como un efecto dañino que debe prohibirse o erradicarse, se "viola su ser sujeto y su dignidad humana" (Liebel, 2006: 109). Con lo cual, luchan por normas que mejoren sus condiciones laborales y les ayuden a trabajar con dignidad.

También es necesario tener en cuenta que el ser sujeto de las organizaciones de los niños no surge sólo del hecho de que los niños estén trabajando y que su trabajo genere un beneficio social, sino que el concepto está influenciado por cuestiones culturales. 
Así, podemos ver que en muchas sociedades es usual que los NNA asuman una responsabilidad a temprana edad desempeñando actividades que, a veces, son pesadas y conllevan un riesgo. Pero éstas son escogidas y dosificadas de tal manera que les permite familiarizarse con ellas y paulatinamente organizarlas a su manera.

Un ejemplo de esto lo obtuvimos cuando muchos NNA entrevistados en Tapachula en el 2009 nos decían que migraban para trabajar porque querían contribuir a la economía familiar, pero también porque "ya era hora de salir" de su hogar. ${ }^{8}$ Culturalmente ya estaban determinados a que en cualquier momento deberían trasladarse al otro lado de la frontera para trabajar, lo cual no les generaba complicaciones al momento de decidirse ,aunque las condiciones de trabajo no fueran las más favorables para ellos. En este sentido, Norberto Liwski considera que "saber cuál ha sido la opinión del niño respecto al desplazamiento decisión que transforma radicalmente su vida- es primordial para poder pensar en forma integral la satisfacción o vulneración de otros derechos." (Liwski, 2008: 3).

De la creación de estos movimientos se desprende la importancia de que los NATs tengan un protagonismo, ya sea espontáneo u organizado. El primero:

(...) aparece cotidianamente, de forma individual y colectiva. Se manifiesta sobre todo en las estrategias de sobrevivencia que los niños inventan en diversas situaciones de vida. El caso más común es el de los niños que viven en la calle y que deben mantenerse por su propia cuenta (Liebel, 2007: 129).

El segundo:

la forma organizada, se entiende cuando los niños establecen una relación solidaria para hacer valer sus intereses y sus derechos. La forma ideal de este protagonismo se encuentra en dirigir ellos mismos los movimientos sociales de los NNA en los que las estructuras y normas se construyan de tal forma que permitan la participación en condiciones de respeto y solidaridad (Liebel, 2007: 129).

\section{Niños, niñas y adolescentes: trabajadores migrantes en Tapachula. Un breve recuento histórico}

La ciudad de Tapachula ha sido objeto de estudio desde la segunda mitad del siglo XIX, debido a que se convirtió en la puerta de entrada para muchos empresarios y trabajadores migrantes provenientes de Guatemala, Alemania, China, Estados Unidos, e Inglaterra, entre otros. Los empresarios fueron atraídos por las facilidades de producción, de mano de obra y de exportación que el gobierno federal de Porfirio Díaz les otorgó para que invirtieran capital económico mediante empresas cafetaleras en la zona, dado que la región contaba con las condiciones de suelo, altura, temperatura, medios de transporte y embarque, necesarias para la producción y la comercialización del café. Tanto la importante producción como la comercialización del grano a nivel nacional e internacional fue lo que, años más tarde, produjo el boom cafetalero en el estado. (Helbig, 1961; García de León, 1999; Benjamín, 1991; Arriola, 1995).

La instauración de las múltiples fincas trajo consigo la llegada de mucha mano de obra procedente de distintos puntos del estado de Chiapas y de Guatemala. Es decir, que se produjo una especie de efecto pushpull (Durang y Massey, 2003), que consiste en la existencia de una oferta de trabajo y unas condiciones económicas y sociales necesarias para generar la posibilidad y la necesidad de ir en busca de tales ofertas: falta de trabajo en su lugar de origen, mejores salarios, pocas restricciones migratorias, etcétera.

La mayor parte de los inmigrantes se establecían en el lugar, sólo de manera temporal, específicamente en los periodos en que la producción requería de más 
mano de obra. Entonces, no era común que se diera una inmigración permanente, ya que en sus lugares de origen aún contaban con tierras cultivables, por lo que la emigración temporal se realizaba sólo para complementar el gasto doméstico y/o religioso. ${ }^{9}$

Desdeentonces, lamigraciónlaboral de guatemaltecos para desempeñarse en actividades agrícolas ha estado presente en la región, sólo que dejaron de dedicarse únicamente al cultivo y la cosecha del café para incursionar en otros productos como plátano, papaya, caña, piña o mango.

En la década de 1980, la dinámica migratoria tuvo una transformación significativa ya que, por los movimientos sociales que se originaron en Guatemala y en Centroamérica en general, la migración hacia México, pero sobre todo hacia Estados Unidos, se incrementó considerablemente. Si bien, a principios de la década, existieron algunas facilidades para ingresar a estos países por la vía legal a través del refugio político, posteriormente las medidas de control migratorio se fueron "restringiendo", por lo que quienes ingresaban al país lo hacían de manera indocumentada.

La mayoría de la población guatemalteca, especialmente los hombres, continúo empleándose en las actividades del campo, probablemente debido a que, como eran campesinos en su lugar de origen, sólo sabían realizar este tipo de labores. Aunque hay quienes dicen - como Casillas y Castillo - que la poca presencia en trabajos urbanos no sólo fue por su especialización agrícola sino por "sentido de sobrevivencia", ya que en las ciudades eran "más fácilmente localizables por las autoridades de migración aquéllos con carácter de indocumentados", por lo que ambos factores restringían la posibilidad de conseguir mejores "oportunidades de trabajo" (Casillas y Castillo, 1989: 382). Sin embargo, poco a poco fueron participando en las actividades dentro de la ciudad, entre las que destacan: albañilería, carpintería, plomería, venta ambulante.

En el caso de las mujeres y los NNA fue distinto, porque desde entonces trabajaban en la ciudad — aún sin documentación - en el comercio ambulante, como empleadas domésticas, sexoservidoras, chicleros y boleros. Estas actividades no requerían mayor especialización. En ese entonces, en los estudios académicos realizados no se daban detalles de sus situaciones y condiciones de trabajo puesto que la prioridad estaba en los trabajadores agrícolas, dado que ahí se encontraba el grueso de la población migrante.

Es hasta 1995 cuando Aura Marina Arriola, en su libro La perla del Soconusco proporciona algunos elementos acerca de las actividades en las que las mujeres y los NNA se estaban empleando: cómo llegaban a la ciudad, con quiénes se relacionaban, cuánto ganaban, los espacios de los que se empezaban a apropiar para su recreación y la percepción que la gente del lugar tenía sobre ellos.

Menciona que las mujeres migrantes de Guatemala, muchas de ellas menores de edad, procedían principalmente de los departamentos fronterizos de San Marcos y Retalhuleu y salían en busca de un trabajo mejor remunerado para contribuir en la economía familiar. Llegaban acompañadas de algún familiar (padres, hermanas, primas) o de amigas del mismo lugar de origen, quienes les ayudaban a conseguir empleo como trabajadoras domésticas los domingos en el parque central Miguel Hidalgo o en las calles del mercado de San Juan y San Sebastián.

Muchas veces los padres no estaban de acuerdo en que vinieran a México en busca de trabajo por los peligros a los que se enfrentaban, pero para ellas no sólo significaba migrar por "necesidad", sino porque la ciudad representaba un "símbolo de confort, de una calidad de vida superior" (Arriola, 1995: 118).

En las entrevistas que la autora presenta en el trabajo muestra que, para ellas, no es tan complicado atravesar la frontera..$^{10}$ Algunas pagaban el pase (50 centavos de peso) y otras eludían dicho pago, ya que la mayoría eran de familia numerosa (algunas tienen hasta 12 hermanos).

Respecto a las condiciones de trabajo, las entrevistadas dijeron que trabajaban de lunes a 
sábado, la mayor parte del día haciendo el aseo, lavando, planchando, algunas cocinando y cuidando niños (pero otras no). El domingo era el día de descanso. Muchas dormían en la casa de trabajo y una que otra en casa de familiares (tías, hermanos). Ganaban entre 150 y 200 pesos mensuales (más que en su país, donde percibían entre 100 y 150 pesos). Esta paga les servía para enviar remesas a sus padres y para comprarse ropa, zapatos y algún accesorio, pero en ningún caso se quejaron de malos tratos por parte de quienes las empleaban. Todas mencionaron que la razón de migrar fue para ayudar a sus familias, aunque reconocieron que estaban en Tapachula porque "les gustó la ciudad", o porque "es bien alegre".

La percepción que tienen los pobladores del lugar sobre ellas en el ámbito laboral está determinada por la condición étnica. La mayoría piensa que "las de tradición indígena" son "honradas y muy trabajadoras", pero a las que llaman "ladinas" les tienen desconfianza, porque con varias de ellas se habían registrado casos de "hurto" en algunos hogares donde trabajaban.

En cuanto a los niños, niñas y adolescentes, la situación laboral era aún más difícil que la de las trabajadoras domésticas, puesto que su lugar de trabajo era la calle y, en muchos casos, también su lugar de residencia. La autora señala que "los niños ${ }^{11}$ pobres y provenientes de Guatemala son la población más vulnerable de la sociedad urbana. (Arriola, 1995: 147).

Este autor hace una diferenciación entre los niños de la calle y los niños en la calle. Los primeros, son aquellos que aún mantienen una relación con sus familiares. En el día, venden en las calles chicles, dulces, cigarros, muñecos típicos de Guatemala, telares, plumas forradas, entre otros artículos, se ofrecen como mandaderos o cargadores y, en las noches, duermen en casa de ellos (hermanos, tíos o algún otro). Los segundos tienen una condición distinta, ya que viven y trabajan en la calle y han roto sus vínculos familiares. Se dedican principalmente a pedir limosna, limpian parabrisas, se pintan de payasitos para ganar dinero en los semáforos, lavan carros. Muchos también se dedican a pedir comida en las casas, calles y restaurantes, a robar alimentos, o a los transeúntes. Generalmente, duermen afuera de los cines, en zaguanes, "en cualquier terminal de autobuses, en las bancas del parque, junto a un árbol, en los quicios de las puertas" (Arriola, 1995: 148). El autor menciona que era cada vez más notorio el abundante número de "niños" en la ciudad y calcula que había alrededor de "100 niños".

La presencia de niños, niñas y adolescentes de Guatemala y otros países de Centroamérica, como Honduras o el Salvador, empleados en diversas actividades, dinamizaba la vida cotidiana en Tapachula. Asimismo, generaba algunas inconformidades entre los lugareños por la apropiación de espacios, especialmente recreativos como el parque central Miguel Hidalgo. Muchos se rehusaban a ir de paseo al parque los domingos porque era ocupado por las trabajadoras domésticas y otros migrantes centroamericanos.

Hasta 1965 el parque había sido punto de reunión de los jóvenes de familias prominentes y de "la clase media" de la ciudad: ahí establecían sus relaciones de amistad y de noviazgo. Después de la "ocupación" de este espacio por parte de la población migrante tuvieron que buscar otros que lo sustituyeran, como los restaurantes de Puerto Madero (hoy Puerto Chiapas), o Playa Linda. Los que tenían menos recursos iban a pasear al parque de Los Cerritos, situado al sur de la ciudad, y otros iban a las albercas públicas cercanas a la frontera. Desde entonces, al parque central sólo acudían para contratar a las trabajadoras domésticas.

Para los niños, niñas y adolescentes migrantes era atractivo asistir al parque central porque, generalmente, llegaban "grupos musicales de rock, rap, tropical, marimba y, algunas veces, danzas folklóricas de las distintas regiones de la república mexicana. El hecho de que fuera el lugar de reunión y recreación para los NNA trabajadores los domingos, poco a poco fue institucionalizándolo como el punto oficial para establecer sus relaciones sociales y laborales. 
Pese a que Arriola se limitó a describir brevemente la situación y condición de los niños migrantes trabajadores en Tapachula, hacía falta mucho por explorar. Fue hasta el año 2006, cuando se realizó uno de los primeros estudios exploratorios que aborda esta problemática, específicamente, las situaciones de trata de mujeres y menores de edad, llevado a cabo por un equipo de trabajo multidisciplinario bajo la coordinación de Rodolfo Casillas.

En el texto La trata de mujeres, adolescentes, niñas y niños en México, señala que hasta la década de 1990, en los estudios sobre migración en esta frontera, no se "hacía referencia a los flujos de mujeres y menores migrantes" a pesar de haberlos visto laborando en diversas actividades agrícolas y de servicios informales, ya que entonces no se les consideraba sujetos de estudio.

El texto se compone de tres partes: la primera, se refiere a las variables que definen el perfil sociodemográfico de ambos grupos de estudio (mujeres y NNA); la segunda, da cuenta de los aspectos de la vida cotidiana en la que se desarrollan los sujetos estudiados, entre otros: alimentación, vivienda, facilidades de movimiento, seguridad, consumo de drogas y alcohol, redes de amistad, relaciones de pareja, sexualidad, maternidad, enfermedades e infecciones de transmisión sexual; y la tercera, se refiere a la vida laboral, en donde se describe a qué se dedican los actores inmersos en la trata de personas, las condiciones laborales, la percepción que tienen de su actividad laboral y las secuelas futuras que podrían producirse como resultado de las actividades que desempeñan.

Como se puede ver, los detalles que presenta Casillas en cada uno de estos apartados son minuciosos en comparación con el trabajo de Arriola. Menciona, entre otros datos: horarios y actividades específicas que realizan como parte de sus labores cotidianamente, así como el aspecto personal (cómo se visten), los alimentos que consumen (que muchas veces no son los mismos que consumen los empleadores), los espacios en los que comen, duermen y se les suele encontrar en horas de trabajo, las relaciones que establecen con los demás (amistad, noviazgo y sexual), los salarios que perciben por sus actividades, las edades entre las que oscilan y, sobre todo, las condiciones de maltrato y discriminación de las que son objeto en el ámbito laboral y fuera de él.

Muchas condiciones de vida y de trabajo en las que se desarrollaban cotidianamente los NNA en la ciudad durante ese año eran muy similares a las planteadas por Arriola. En este caso se trató de describir de manera más detallada cada una de esas condiciones y de enfatizar que, a pesar de que era cada vez mayor la presencia de migrantes menores de edad, las autoridades mexicanas y las de sus países de origen no trabajaban en favor de proponer una regulación constitucional efectiva que contribuyera a evitar el abuso y maltrato a dicha población. Lo destacable de este trabajo es que expone las malas condiciones en las que viven y trabajan los NNA migrantes, pero se queda solamente en la emisión de recomendaciones a las autoridades correspondientes, quienes tienen la decisión de tomarlas en cuenta, desecharlas o simplemente ignorarlas, dependiendo de su injerencia en las políticas públicas, en la legislación de nuevos estatutos o de reformas jurídicas.

Además de este estudio, se realizaron otros en torno a los derechos humanos de aquellos que se quedan establecidos temporal o definitivamente en la frontera sur así como de los transmigrantes, es decir, los que van de paso por el territorio mexicano para llegar a Estados Unidos (PDH, 2005; Escobar, 2008; Nájera, 2011, entre otros).

En el año 2009 se hizo un nuevo estudio sobre los NNA migrantes en Tapachula, titulado Niños, niñas y adolescentes migrantes centroamericanos en la frontera sur de México ${ }^{12}$, en el cual tuve la oportunidad de coordinar el equipo de trabajo de campo y escribir, casi en su totalidad, el informe final.

El objetivo no era únicamente ver los cambios y las continuidades en cuanto a las situaciones y condiciones 
de vida de esta población centroamericana — sobre todo de los que trabajaban en la calle bajo distintas modalidades (ambulantes, establecidos en puntos específicos en espacios públicos y bajo techo) y las empleadas domésticas - sino explorar lo que sucedía alrededor de los que eran asegurados en cualquier parte del país y trasladados a los albergues de esta ciudad fronteriza, así como los que se empleaban en el trabajo agrícola. La información obtenida en cada una de las categorías abordadas fue variada, aunque en las dos primeras se presentaron más elementos descriptivos.

Entre los puntos más sobresalientes encontramos que en ese momento la mayoría de la población infantil que estaba trabajando en las distintas actividades dentro de la ciudad provenía principalmente de Guatemala. Durante los dos meses de trabajo de campo no se encontró a nadie de otro país centroamericano. Algunas actividades estaban delimitadas por el género, es decir, sólo los hombres se desempeñaban como boleros y chicleros, ${ }^{13}$ mientras las mujeres estaban destinadas principalmente al trabajo doméstico. ${ }^{14}$

También era notorioquelacantidad deestapoblación en las calles había disminuido considerablemente respecto a años anteriores, ya que se tenía como punto de referencia el trabajo de Rodolfo Casillas sobre el tema de la trata laboral publicado en el 2006, además de la percepción de los mismos entrevistados. Sin embargo, hubo quienes aseguraban que esta situación seguía siendo igual que en años anteriores.

Algunos argumentos que justificaban esta reducción de migrantes (tanto de menores de edad como de adultos), eran que este lugar dejó de ser punto de partida del tren, por lo que Tapachula se convirtió sólo en ciudad de paso, ya que tenían que abordarlo hasta Arriaga. Incluso se comenzó a ver que los migrantes buscaban nuevas rutas para evitar pasar por la ciudad. Otras razones eran que estaban migrando hacia distintos puntos del estado e incluso del país y que los niños, niñas y adolescentes estaban siendo constantemente hostigados por trabajar en las calles y parques. Cuando llegaban los cruceros al Puerto Chiapas era evidente la "limpieza" que se hacía en la ciudad desde la madrugada. Los trabajadores eran desplazados hacia sitios en los que no fueran tan visibles, por ejemplo, en los alrededores del mercado y los que deambulaban por las calles eran presionados para que no se detuvieran mucho tiempo en un sólo lugar.

Aura Marina Arriola menciona en su estudio que el parque Miguel Hidalgo continuaba siendo el centro de reunión de los NNA trabajadores migrantes que acudían el día domingo para recrearse y para establecer todo tipo de relaciones, pero también era el espacio de trabajo durante toda la semana.

Como el parque había dejado de ser un espacio recreativo para los lugareños desde hacía mucho tiempo, los gobiernos estatal y local construyeron uno nuevo en 2008, conocido como parque Bicentenario. A quienes intentaban atravesarlo para pasar a vender $u$ ofrecer algún servicio (chicleros, boleros, empleadas domésticas) se les prohibía el pasó, ${ }^{15}$ así como a los que sólo querían cambiar de ambiente para sentarse a platicar o caminar alrededor del mismo.

La diferencia entre uno y otro era notable. Por ejemplo, el parque Bicentenario, totalmente iluminado, tiene fuentes de agua a las que acuden los niños y adultos para sofocar el calor. Regularmente, llegaban al kiosko grupos musicales que amenizaban las tardes-noches con piezas de marimba, mientras algunos bailaban. En el parque Miguel Hidalgo había espacios que tenían poca iluminación y, durante el tiempo que duró el trabajo de campo, no se observó que en algún domingo realizaran algún evento musical, artístico u otro tipo de entretenimiento para los visitantes. También llegaban grupos de personas de variadas adscripciones religiosas a predicar, promover la asistencia a sus iglesias o, en tiempos electorales, para hacer mítines de campañas políticas.

Las condiciones laborales seguían siendo muy similares a las que Aura Marina registró ya que continuaban teniendo largas jornadas laborales: 
regularmente de 7 am. a 10 pm. Sus salarios eran de 50 pesos diarios y vivían con familiares, con sus empleadores o rentaban habitaciones individual o colectivamente (de 2 hasta 10 niños) pagando entre 400 y 600 pesos por mes. Unos comían en casa de sus empleadores, en pequeños establecimientos llamados cocinas económicas (el costo por comida era de 20 pesos, aunque podían venderles la mitad de la ración). Otros compraban tortillas que acompañaban con agua tomada de alguna llave en la vía pública y otros simplemente no comían. Cabe decir que muchos sólo consumían alimentos una o dos veces al día y se recreaban en el parque el día domingo, aunque había quienes no tenían ni día ni hora asignada para esto, así que jugaban con sus espacios y horarios de trabajo. ${ }^{16}$

En el plano jurídico también hubo avances. Por iniciativa del gobernador del Estado de Chiapas, Juan Sabines Guerrero, y avalado por el Congreso Estatal y el gobierno federal, se promovió la creación de instituciones estatales, algunas de ellas dependientes de la PGR, con la finalidad de atender la violencia ejercida en contra de esta población. ${ }^{17}$ Entre otras: la Comisión Estatal para la Protección de los Derechos humanos de los Migrantes, la Fiscalía Especializada en Atención a Delitos Cometidos en Contra de Inmigrantes, el albergue especial para víctimas de trata de FEVIMTRA, agencias del ministerio público en Tapachula y Huixtla adscritas a la Fiscalía Especializada en Atención a Delitos Cometidos en Contra de Inmigrantes y la Secretaría para el Desarrollo de la Frontera Sur.

En el informe final del proyecto de 2009, las autoras del texto planteamos en las conclusiones que, a pesar de que las distintas instituciones encargadas de cuidar los derechos de la niñez migrante han mostrado avances significativos en cuanto a la promoción de nuevas leyes y la reformulación de otras para combatir los abusos que se comenten contra ellos, aún queda un largo camino por recorrer. Si bien se han creado organismos estatales e incrementado la participación con otros ya existentes para dicho fin, era evidente la falta de una coordinación multilateral en la que trabajasen de manera conjunta las que dependen del gobierno mexicano, las de los gobiernos centroamericanos, así como las organizaciones civiles y sociales nacionales e internacionales.

Esta desarticulación y, en algunos casos, la falta de compromiso para que las estrategias planteadas no obtuvieran los resultados que deberían, tanto en la prevención como en la resolución de problemas a los que los NNA se enfrentaban cotidianamente. Asimismo, no parece haber un vínculo estrecho entre las instituciones, la niñez migrante y la sociedad civil, ya que de otra forma podría verse reflejado en los NNA, un ligero conocimiento sobre sus derechos y obligaciones como trabajadores. De ahí que estén expuestos a una serie de abusos por parte de la población local que los emplea, (que pueden ser mexicanos o sus connacionales) y a una mayor sensibilidad por parte de la población local hacia ellos.

Con esta breve descripción etnográfica se pretendió dar cuenta de la presencia de los niños, niñas y adolescentes trabajadores migrantes a lo largo de aproximadamente tres décadas (1980-2010) en la ciudad de Tapachula. Se dejaron entrever algunos cambios y continuidades en cuanto a las condiciones laborales y de vida en las que se desenvuelve esta población. Sin embargo, es necesario que estos datos sean reflexionados a la luz de algunos conceptos que permitan entender un poco más la dinámica de la niñez y la adolescencia trabajadora en Tapachula.

\section{Consideraciones finales}

El dato etnográfico presentado en el apartado anterior revela que las disposiciones bajo las cuales la OIT y la UNICEF se oponen al trabajo de los NNA queda fuera de la realidad. Podemos ver que desde hace varias décadas este sector de la población guatemalteca ha estado en constante migración hacia la ciudad en busca de empleos mejor remunerados que les permitieran 
solventar su situación de vida y contribuir en el gasto doméstico. Pero también es evidente que las condiciones laborales y de vida en las que se desenvuelven en la ciudad no son las más adecuadas, ya que se desarrollan en un ambiente de extrema pobreza, marginación, violencia y discriminación por parte de sus empleadores y de la población local. De hecho, se infringe la edad mínima para trabajar, se afecta su integridad física con arduas jornadas de trabajo (regularmente entre 12 y 14 horas diarias, lo que les impide asistir a la escuela). Es decir, que en muchos sentidos no se atienden las disposiciones planteadas, que esas instituciones tanto defienden, pero que en la práctica están lejos de cumplir.

En este sentido, valdría la pena considerar con mayor atención la propuesta hecha por Cussiánovich y Liebel, en cuanto a que el trabajo de los NNA sea "socialmente aceptado", valorado y respetado y en cuanto a que la organización y participación de los NNA sea primordial para la creación de nuevas formas de trabajo. Aunque el ideal es que no trabajen bajo ninguna modalidad para no afectar su proceso de crecimiento físico, social y psicológico, el hecho es que hasta ahora eso ha sido inevitable, por lo que al menos se debería procurar que lo hicieran en condiciones más óptimas, de manera organizada y participativa, tomando en cuenta su derecho a decidir sobre esas condiciones de manera informada y consciente. En varias de las entrevistas realizadas en el 2009, los NNA expresaron que, en parte, era decisión propia querer trabajar porque les permitía ganar su propio dinero, porque ayudaban a su familia, o porque podían vivir fuera de su comunidad, entre otras razones. No obstante, desconocían que tienen derechos aun siendo indocumentados y que existen instituciones que deben velar por sus condiciones laborales.

Por otrolado, en elámbitojurídico, se han presentado avances en relación a las medidas que contribuyan a evitar la explotación laboral y sexual de los NNA y sobre las formas de castigo para aquellos que la fomentan y la ejercen. Pero no podemos obviar que también se experimenta una simulación de la legalidad (Comaroff,
2009), ya que, aunque existen más leyes que regulan dichas prácticas e instituciones que se encargan de vigilar que éstas se cumplan, en la vida cotidiana, es posible ver cómo las autoridades simulan aplicar las leyes y perpetúan ciertas prácticas que vulneran su condición como individuos.

En este sentido, no se discute el hecho de que los NNA son violentados de múltiples formas en la vida diaria como se ha descrito en los distintos estudios realizados en la ciudad. Sin embargo, es necesario tener más cuidado al momento de emitir juicios respecto a la situación, dado que es común caer en posicionamientos un tanto paternalistas y desesperanzadores ${ }^{18}$ que, lejos de contribuir a la creación de nuevas acciones facilitadoreas de avances significativos en esta materia, lo único que llegan es a plantear recomendaciones que muchas veces se quedan en el tintero. Por otra parte, se tiende a invisibilizar la capacidad de reacción que los NNA tienen ante las múltiples situaciones adversas que enfrentan cotidianamente y las estrategias que emplean para evitarlas o, por lo menos, contrarrestarlas.

Por ejemplo, el artículo escrito por Soledad Álvarez, A la sombra del Miguel Hidalgo: análisis etnográfico del parque central de Tapachula, es el más claro ejemplo de victimización hacia los migrantes centroamericanos en general, y específicamente, hacia los NNA. La razón por la que hago referencia a este texto y no a otros es porque las fechas en que ella hizo trabajo de campo coinciden con los meses que estuve también en la zona. Parecía que estábamos viendo dos ciudades distintas. Ella asegura que el parque Miguel Hidalgo es un espacio donde se explota sexual y laboralmente a los migrantes y se trafica con ellos dando la impresión de que son incapaces de reaccionar ante tales situaciones ${ }^{19}$ donde, además se produce y reproduce la violencia y se hace visible la vulnerabilidad a la que están expuestos. Todo ello a la vista de las autoridades que no se atreven a actuar en contra de esos actos de ilegalidad. 
En contraste, nosotros estábamos viendo un espacio donde se oferta y demanda trabajo donde los NNA crean y reproducen redes sociales para cuidarse entre su círculo de amistades y desarrollan estrategias para evitar ser víctimas de cualquier abuso laboral, donde establecen contacto con familiares y/o amigos para comentar lo sucedido en la semana, donde consiguen atraer las miradas de los demás para entablar relaciones de noviazgo, donde se aprenden y adoptan nuevas expresiones culturales, tales como la forma de saludar entre su círculo más próximo y los demás, formas de vestir, de peinarse, los gustos musicales (desde banda hasta canciones de moda en inglés), etc. El parque era el lugar donde encontraban el estado de liminalidad —en términos de Turner (1993) — que esperaban durante toda la semana.

Si bien lo que Soledad describe en su texto es una parte de la realidad que se vive tanto en el parque como en distintos sitios de la ciudad, también es cierto que no plantea la capacidad de agencia que los NNA migrantes tienen, ni las acciones que emprenden en ese mismo espacio ante las adversidades que se les presentan. Adversidades que les permiten, en la medida de sus posibilidades, enfrentar la violencia y vulnerabilidad a la que están constantemente expuestos.

Por otra parte, las autoridades gubernamentales (consulados, autoridades locales, burócratas de organismos internacionales) y algunos encargados de asociaciones civiles (encargados de instituciones de Derechos Humanos y de albergues, principalmente) son quienes más reproducen acciones y discursos de proteccionismo y de asistencialismo, pero sin un impacto significativo.

Por ejemplo, desde los consulados aseguraban estar trabajando continuamente para mejorar las condiciones de los NNA migrantes que vienen a trabajar a la ciudad. Sin embargo, en la práctica, sólo se traduce en detectar algunos casos de trata sexual y laboral, hacer el seguimiento legal del castigo a esas actividades y a la posterior deportación de la niñez y adolescencia a sus países de origen. Pero no hacían un trabajo desde la base con los principales afectados. Regularmente tomaban medidas como la instalación de albergues que, si bien contribuyeron a solventar las dificultades más inmediatas (como tener alimentos, implementar talleres de manualidades, espacios recreativos o apoyos médicos y psicológicos), se quedaban en el mero asistencialismo - o como dice Cussiánovich, salvacionismo - sin atacar el problema de fondo, de modo que los niños, niñas y adolescentes tomaran el papel protagonista para proponer marcos jurídicos pertinentes y programas sociales desde los que pudieran participar activamente y les permitieran mejorar sus condiciones laborales y de vida. .

No podemos obviar la responsabilidad que los académicos tienen en la producción y reproducción de estos discursos proteccionistas. Muchos de ellos, continúan elaborando y desarrollando proyectos para las diferentes instituciones, sobre todo gubernamentales, porque de ellas obtienen recursos para financiarse cuyos resultados, por ser similares a los obtenidos en estudios anteriores, dan los argumentos suficientes para la repetición de los mismos discursos. De esta manera, también vemos que "lo social pasa a ser un elemento de mercado: los problemas sociales son problemas de mercado. ¿Quién vende mejor el problema de los chicos de la calle, de los infractores de la ley, de los adictos a las drogas en proceso de recuperación?" (Cussiánovich, 2002: 7).

Con esto no quiero decir que los estudios realizados no hayan sido de fundamental importancia para conocer y entender el fenómeno migratorio y laboral de los niños, niñas y adolescentes centroamericanos en México, específicamente en la frontera sur, sino que, según mi opinión, es tiempo de empezar a observar este fenómeno de manera distinta.

Quizás habría que dejar de victimizarlos, de considerarlos sólo como objetos de cuidado a quienes hay que asistir frente a las distintas áreas de violencia 
y vulnerabilidad a las que están expuestos en la ciudad, y sería necesario tomar nuevas actitudes y acciones que promuevan y generen la participación y protagonismo de los NNA. Así se podrían lograr más avances, no sólo en los ámbitos de la violación a sus derechos humanos y de la marginalidad en las que se les ha ubicado, sino también en su aspecto personal —entendido como la confianza en sí mismos-, en la manera de enfrentar los retos que se les presenten día a día.

Es muy probable que muchos NNA migrantes no quieran ser parte de la movilización y la participación en favor de sus derechos ni tampoco que esto sea lo que los libere de los abusos y la explotación laboral y sexual; sin embargo, considero que podría ser un buen comienzo que contribuiría a mejorar las condiciones de este sector de la población.

Sin embargo, es probable que muchos niños, niñas $\mathrm{y}$ adolescentes trabajadores migrantes establecidos en la frontera sur de México aún sigan esperando contar con más alternativas.

\section{Notas}

${ }^{1}$ Entiendo por niñez la conjunción del término niños y niñas.

2 Según Portes y Rumbaut (2006) está definido por el lugar huésped, es decir, es aquella persona que se desplaza de su lugar de origen hacia otro de destino

3 Entiendo la frontera como un "escenario desterritorializado [poroso], en donde las culturas y las identidades son creativamente reinventadas como complejas y multidimensionales formas de referencia" (Garduño, 2003: 66).

${ }^{4}$ Convención sobre los Derechos del Niño, adoptada y abierta a la firma y ratificación por la Asamblea General en su resolución 44/25, de 20 de noviembre de 1989. Entrada en vigor: 2 de septiembre de 1990, de conformidad con el artículo 49.

5 Ver en la página: http://www.diputados.gob.mx/ LeyesBiblio/pdf/185.pdf. De esta misma manera concibe la diferencia entre niños, niñas y adolescentes la Organización Internacional del Trabajo (OIT).

6 Ver página 〈http://www.ilo.org/ipec/facts/lang--es/ index.htm> [28 de agosto de 2011]

7 Ver página 〈http://www.mflor.mx/materias/temas/ einfantil/einfantil.htm> [28 de agosto de 2011]

$8 \mathrm{Si}$ bien es cierto que la necesidad económica, la reunificación familiar y la inseguridad producto de la violencia en sus países de origen son las principales causas de la migración, no podemos obviar que existen otros aspectos que impulsan la migración: porque "es hora de migrar", por independencia, por "conocer otros lugares", etc. Migrar les permite alcanzar una mayor madurez y en el hogar adoptan nuevos roles, como puede ser el de proveedores económicamente.

${ }^{9}$ En el caso de los inmigrantes chiapanecos, la mayoría provenía de la zona Altos, principalmente de los municipios de Chamula, Zinacantán y Oxchúc. Existen fuentes escritas que dan cuenta de que uno de los principales motivos por el que los pobladores (hombres) emigraban hacia el Soconusco era reunir el suficiente dinero que les permitiera sufragar los cuantiosos gastos de las fiestas patronales cuando eran elegidos para desempeñar algún cargo religioso. (Pozas, 1952; García de León, 1998; Wasserstrom, 1989, entre otros).

${ }^{10}$ En este sentido, también apuntaba que percibía lo contradictorio de los controles migratorios en esta frontera, ya que eran "por un lado, muy rigurosos y violentos con los transmigrantes $y$, por el otro, muy permisivos con la mano de obra centroamericana que es indispensable desde la Colonia para la acumulación de capital en la región" (Arriola, 1995: 118).

"Incluye en la categoría de "niños" a las niñas y adolescentes de ambos sexos.

${ }^{12}$ Éste es el título del informe final que entregamos al término del proyecto de investigación. El estudio fue financiado por la Organización Internacional para las Migraciones(OIM) y el responsablefue Rodolfo Casillas R.. Pero el equipo de trabajo de campo -integrado por Magnolia Gómez Domínguez, David Sibaja Vargas, 
Francisco Aceves Verdugo y Yasmina A. López Reyes (quien coordinó dicho equipo) - fue el que se encargó del levantamiento de datos. Asimismo, el informe final fue redactado por Yasmina López y Magnolia Gómez y publicado con el nombre del responsable del proyecto, pese al previo acuerdo de coautoría que éste tenía con la coordinadora de equipo. La misma versión aparece en una publicación hecha por la Comisión de Derechos Humanos y la Organización Internacional para las Migraciones en el 2009 (cuya página ha sido dada de baja de la red) http://www.cndh.org.mx/ publica/libreria/varios/NI\%C3\%9lO-AS-ADOLESMIGRANTES.pdf [18 de junio de 2011]) y, en el 2010, apareció publicada una nueva versión del informe con el nombre Vidas en pañales. Niños, niñas y adolescentes en poblaciones del sur de México http://www.oim.org.mx/ mini_s/estudio trata2010/docs/trata estudio 2010. pdf [18 de junio de 2011]. Quiero precisar que las citas textuales que aparecen en este artículo se tomaron directamente del informe de campo que mi compañera y yo entregamos el 20 de septiembre de 2009 a Rodolfo Casillas, en el que ya estaban incluidos los comentarios hechos por la Comisión Dictaminadora de la OIM, por lo que no lo citaré bajo el nombre de quien se adjudicó dicha autoría.

${ }^{13}$ En el mes de julio de 2011, mientras hacía un recorrido de campo, me pude percatar de que ya había varias mujeres adolescentes y adultas desempeñando esta actividad, mientras que en el 2009, sólo se manifestó el caso de una mujer adulta.

${ }^{14}$ Sobre el papel que juegan las mujeres migrantes como empleadas domésticas en la ciudad, ver el trabajo de Mercedes Olivera citado en la bibliografía.

15 Comúnmente se encontraban uno o dos policías municipales resguardando las esquinas del parque.

16 Por ejemplo, a los chicleros, boleros, floristas, vendedores de algodón y globos se les veía jugar con otros compañeros en las calles, ya sea que estuvieran en movimiento por las calles o en sus puntos de trabajo establecidos.
${ }^{17}$ Además de promover su imagen pública dentro de la política internacional.

${ }^{18}$ En el que me incluyo, por lo que se alcanza a leer en varias partes del informe que escribí en el 2009. Sin embargo, quiero decir que entonces me era difícil tener un posicionamiento más crítico porque era la primera vez que me enfrentaba a este tipo de realidades y desconocía totalmente la discusión académica sobre el tema.

${ }^{19}$ Algo semejante expresa Carolina Escobar: "Debido a la inmadurez propia de su edad y a su corta experiencia de vida (aunque sus experiencias hayan sido fuertes), la mayoría de ellos están casi indefensos ante los desafíos que les plantea la migración" (Escobar, 2006: 59).

\section{Bibliografía}

Arriola, Aura Marina (1995), "Tapachula. 'La perla del Soconusco'. Ciudad estratégica para la redefinición de las fronteras, Guatemala: Flacso-Guatemala-DEASINAH.

Álvarez Velasco, Soledad (2010), "A la sombra del Miguel Hidalgo: análisis etnográfico del parque central de Tapachula”, en Revista Liminar. Estudios Sociales y Humanísticos, vol. VIII, núm. 2, diciembre. México: Universidad de Ciencias y Artes de Chiapas, Pp. 129152.

Benjamín, Thomas Louis (1990), Camino a Leviatán, México: Consejo Nacional para la Cultura y las Artes (Colección Regiones).

Bronfman, Mario; René Leyva y Mirka Negroni (2004), Movilidad poblacional y VIH/SIDA. Contextos de vulnerabilidad en México y Centroamérica, México: Instituto Nacional de Salud Pública.

Casillas, Rodolfo (2006) La trata de mujeres, adolescentes, niñas y niños en México: un estudio exploratorio en Tapachula, Chiapas, México: Comisión Interamericana de Mujeres/Organización de Estados Americanos/ Organización Internacional para las Migraciones/ Instituto Nacional de las Mujeres/Instituto Nacional 
de Migración. http://www.oas.org/atip/Reports/ Estudio.Exploratorio.en.Tapachula.pdf > [21 de junio de 2009].

Casillas, Rodolfo y Miguel Ángel Castillo (1989), "Mitos y realidades sobre las migraciones centroamericanas a Chiapas", en Luis Hernández Palacios y Juan Manuel Sandoval, (comp.), El redescubrimiento de la frontera sur, México: Anciem Régime, pp. 373-390.

Comaroff, Jean y John Comaroff (2009), Violencia y Ley en la Pos-colonia: una reflexión sobre las complicidades NorteSur. Katz Editores, Buenos Aires.

Cussiánovich Villarán, Alejandro (2002), “Tipología del trabajo infantil desde el punto de vista de los derechos humanos: la necesidad de una diferenciación." Conferencia Internacional: Acabar con la explotación económica del niño y la imposición de los derechos humanos a través de nuevos enfoques en la lucha contra la pobreza, Alemania. http://www.woekweb. de/web/cms/upload/pdf/forum_kinderarbeit/ publikationen/cussianovich_2002_tipologia_del_ trabajoinfantil.pdf, [consultado: 5 de agosto de 2011].

Durand, Jorge y Douglas S. Massey (2003), Clandestinos. Migración México-Estados-Unidos en los Albores del Siglo XXI. Universidad Autónoma de Zacatecas/Porrúa, México.

Escobar Sarti, Carolina (2008), Los pequeños pasos en un camino minado. Migración, niñez y juventud en Centroamérica y el sur de México, Guatemala: Consejería en Proyectos. http:/www.pcslatin.org/drupal/files/ LibroPequenospasos.pdf [13 de julio de 2011].

García de León, Antonio (1998), Resistencia y utopía. Memorial de agravios y crónica de revueltas y profecías acaecidas en la provincia de Chiapas durante los últimos quinientos años de su historia. México: ERA.

Garduño, Everardo (2003), “Antropología de la Frontera, la Migración y los Procesos Transnacionales.” En Frontera Norte, Vol. 15, Julio-Diciembre, El Colegio de la Frontera Norte, Tijuana. Pp. 65-89.

Helbig, Carlos (1961), El Soconusco y su zona cafetalera en Chiapas. Chiapas: Instituto de Ciencias y Artes.
Informe final de trabajo de campo del proyecto "Niños, niñas y adolescentes migrantes centroamericanos en la frontera sur de México", financiado por la Organización Internacional para las Migraciones y redactado por Yasmina A. López Reyes y Magnolia Gómez Domínguez, 20 de septiembre de 2009, San Cristóbal de Las Casas, Chiapas.

Kauffer Michel, Edith F.; Jan De Vos, Gabriela Patricia Robledo Hernández (Edits.) (2002), Identidades, Migraciones y Género en la Frontera Sur de México, México: El Colegio De La Frontera Sur.

Liebel, Manfred (2006), "Los movimientos de niños, niñas y adolescentes trabajadores. Un enfoque desde la sociología”, en Revista Política y Sociedad, Vol. 43, Núm. 1. Madrid: Universidad Complutense de Madrid, Pp. 105-123. http://revistas.ucm.es/index. php/POSO/article/view/POSO0606130105A/22678 [21 de agosto de 2011).

Liebel, Manfred (2007), "Paternalismo, participación y protagonismo infantil" en Yolanda Corona C. y María E. Linares (coord.) Participación infantil y juvenil en América Latina, México: UAM. Pp. 113-146. También disponible en http://www.sename.cl/wsename/ otros/Paternalismo_manfred_liebel.pdf, [consultado: 21 de agosto de 2001].

Liwski, Norberto (2008), Migraciones de niñas, niños $y$ adolescentes bajo el enfoque de derechos. Instituto Interamericano del Niño, la Niña y Adolescentes, OEA. http://www.iin.oea.org/iin/cad/actualizacion/ pdf/3_l/Migraciones $\% 20 d e \% 20 \mathrm{Ni} \% C 3 \%$ Blos $\% 20$ $\mathrm{Ni} \%$ C3\%Blas\%20y\%20Adolescentes\%20bajo\%20 el\%20Enfoque\%20de\%20Derechos.pdf [17 de julio de 2011].

Nájera Aguirre, Jessica N. (2011), Nuevos acuerdos familiares entre los miembros de las familias guatemaltecas ante la movilidad laboral transfronteriza a México, a partir de la crisis agrícola y la terciarización económica chiapaneca, Ponencia presentada en el IV Seminario Internacional sobre Familia. Desafíos para comprender los procesos de la migración internacional y las familias. Universidad 
de Caldas, Manizales, Colombia, del 25 al 29 de abril. http://estudiosdefamilia.co/pdfs/Jessica\%20Najera. pdf, [consultado: 14 de julio de 2011).

Olivera Bustamante, Mercedes y Luis Antonio Sánchez Trujillo (2008), "Género: iestructura estructurante de la migración? En Daniel Villafuerte Solís y María del Carmen García Aguilar (coords.). Migraciones en el sur de México y Centroamérica. México: Universidad de Ciencias y Artes de Chiapas-Miguel Ángel Porrúa, Pp. 247-274.

Pico Merchán, María Eugenia y Miriam Salazar Henao (2008), "El trabajo infantil como práctica de crianza: contexto de una plaza de mercado", en Revista Hacia la Promoción de la salud, Volumen 13. Colombia: Universidad de Caldas, Pp. 95-120, http://promocionsalud.ucaldas.edu.co/downloads/ Revistal3_6.pdf, [consultado: 21 de julio de 2011].

Procurador de Derechos Humanos (PDH) (2005), Derribando muros. La realidad de la niñez y adolescencia migrante en la frontera Guatemala-México. Guatemala: Defensoría de la niñez y juventud. http://imprasc.
net:29572/Perfilesnacionales/Documents/Guatemala/ D02.pdf [17 de julio de 2011].

Rivas Castillo, Jaime (2008), Tejiendo redes frente al riesgo y la vulnerabilidad: migrantes centroamericanos y organizaciones civiles de apoyo en Tapachula, Chiapas, Tesis de maestría en Antropología Social, Centro de Investigaciones y Estudios Superiores en Antropología Social (CIESASSureste), San Cristóbal de las Casas, Chiapas, México.

Turner, Víctor (1993), "Pasos, márgenes y pobreza: símbolos religiosos de la Communitas", en Paul Bohannan (et. al.) Antropología Lecturas, Mc GrawHill. Pp.515-544.

Villafuerte Solís, Daniel y María del Carmen García Aguilar (coord.) (2008), Migraciones en el sur de México y Centroamérica. México: Universidad de Ciencias y Artes de Chiapas, Miguel Ángel Porrúa.

Pozas, Ricardo (1952), Juan Pérez Jolote, Mexico: Fondo de Cultura Económica-Instituto Interamericano Indigenista.

Wasserstrom, Robert (1989), Clase y sociedad en el centro de Chiapas. México: Fondo de Cultura Económica. 